\title{
Caractéristiques de l'élevage du canard de Barbarie dans les zones agro-écologiques du Sud-Bénin
}

\author{
Finagnon Josée Bernice HOUESSIONON ${ }^{*}$, Gabriel Assouan BONOU1, 5, Gbénagnon Serge \\ AHOUNOU', Mahamadou DAHOUDA2, Tossou Jacques DOUGNON³, Guy Apollinaire MENSAH4, \\ Sallou BANI KOGUI', Issaka YOUSSAO ABDOU KARIM 1*
}

${ }^{1}$ Laboratoire de Biotechnologie Animale et de la technologie des viandes, Département de production et Santé Animales, Ecole Polytechnique d'Abomey-Calavi, Université d'Abomey-Calavi, 01 BP 2009, Cotonou, République du Bénin;

2Faculté des sciences agronomiques, Université d'Abomey-Calavi, République du Bénin;

${ }^{3}$ Laboratoire de recherches en Biologie appliquée, Ecole Polytechnique d'Abomey-Calavi, Université d'AbomeyCalavi, 01 BP 2009, Cotonou, République du Bénin;

${ }^{4}$ Centre de Recherche Agricole d'Agonkanmey, Institut Nationale de Recherche Agricole du Bénin, 01 BP 884, Cotonou 01, République du Bénin.

${ }^{5}$ Centre de Recherche Agricole du Nord, Institut Nationale de Recherche Agricole du Bénin, 01 BP 884, Cotonou 01, République du Bénin.

*Corresponding author E-mail: ⿺houessionon@yahoo.fr,_Numéro de téléphone: 0022997767071 or 0022965640859

Original submitted in on $2^{\text {nd }}$ October 2019. Published online at www.m.elewa.org/journals/ on 31 st January 2020 https://doi.org/10.35759/JABs.145.3

\section{RESUME}

Objectif : L'élevage du canard de Barbarie est moins développé au Bénin comparativement aux poulets et à la pintade. L'objectif de l'étude est de caractériser cet élevage dans le Sud du Bénin.

Méthodologie et résultats : Ainsi, une enquête a été réalisée de Juin 2015 à Août 2016 dans 213 élevages de canard de Barbarie répartis dans trois zones agro-écologiques: dépression, pêcheries et terres de barre. L'élevage de canard de Barbarie est pratiqué par plusieurs catégories socio-professionnelles dont l'âge varie de 39 à 42ans. II est plus pratiqué par les hommes dans les zones des pêcheries $(67,86 \%)$ et des terres de barre $(78,50 \%)$ et plus par les femmes $(59,55 \%)$ dans la zone de dépression $(p<0,05)$. Les éleveurs de canard sont majoritairement non scolarisés et la plupart des élevages ont été créés entre 2000 et 2016. La proportion des aviculteurs qui élèvent les canards de Barbarie en divagation dans la zone de dépression $(90,91 \%)$ est significativement plus élevée $(p<0,05)$ que celles des zones des pêcheries $(52,38$ $\%$ ) et des terres de barre $(63,55 \%)$. Les éleveurs qui distribuent des céréales, des sous-produits agricoles et des restes de cuisine aux canards dans la zone de dépression (100\%) et dans la zone des pêcheries $(95,24 \%)$ sont plus fréquents $(p<0,05)$ que ceux de la zone des terres de barre $(69,16 \%)$. La fréquence des éleveurs de la zone de dépression $(31,82 \%)$ qui font l'élevage des canards de Barbarie pour la vente et l'auto consommation est inférieure $(p<0,05)$ à celles des zones des pêcheries $(57,14 \%)$ et des terres de barre $(67,29 \%)$. Dans la zone des terres de barre, la proportion des éleveurs $(63,21 \%)$ qui soigne les canards est supérieure $(p<0,05)$ à celle des zones des pêcheries $(46,43 \%)$ et de dépression $(36,36 \%)$. A 
l'exception de l'alimentation, les canards ne sont pas exigeants pour l'habitat; ils croissent mieux et résistent plus aux pathologies comparativement aux poulets dans les trois zones de l'étude.

Conclusion et application: L'élevage des canards de Barbarie dans le Sud du Bénin est de type familial avec une diversité observée dans le mode d'élevage, l'alimentation et le suivi sanitaire dans les différentes zones agro écologiques. Les résultats contribueront à la mise en place d'un programme d'amélioration des systèmes d'élevage.

Mots clés : Elevage ; Canard de Barbarie ; Zone agro-écologique ; Bénin

\section{ABSTRACT}

Background and objective: Muscovy duck breeding is less developed in Benin compared to chickens and guinea fowl. The study aimed to characterize this breeding in South-Benin.

Methodology and results: A survey was carried out from June 2015 to August 2016 in 213 Muscovy duck farms in three agro-ecological zones: depression, fisheries and bar lands. The Muscovy duck breeding is practiced by several socio-economic professional categories whose age varies from 39 to 42 years. It is more practiced by men in fisheries (67.86\%) and bar lands (78.50\%) zones and more by women $(59.55 \%)$ in depression zone $(p<0.05)$. Duck farmers are largely out-of-school persons and most of the farms were created between 2000 and 2016. The proportion of poultry producers who breeds Muscovy ducks in free range in depression zone $(90.91 \%)$ is significantly higher $(p<0.05)$ than those in fisheries $(52.38 \%)$ and bar lands $(63.55 \%)$ zones. Breeders giving cereals, by-products and kitchen leftovers in depression zone $(100 \%)$ and in fishery zone $(95.24 \%)$ are more frequent $(p<0.05)$ than those in bar lands area $(69.16 \%)$. The frequency of breeders in depression zone (31.82\%) who rear Muscovy ducks for sale and selfconsumption is lower $(p<0.05)$ than those in fisheries $(57.14 \%)$ and in bar lands $(67.29 \%)$ zones. In bar lands zone, the proportion of breeders $(63.21 \%)$ who gives ducks medical care is higher $(p<0.05)$ than that of fisheries (46.43\%) and depression (36.36\%) zones. Frequencies of breeders treating ducks by selfmedication in bar lands $(50.47 \%)$ and fisheries $(40.24 \%)$ zones are higher $(p<0.05)$ than that of depression (4.55\%). A part from feeding, ducks are not exigent for habitat; they grow better and are more resistant to pathologies compared to chickens in the three study areas.

Conclusion and application: The Muscovy duck breeding in the three agro-ecological zones is of family type with a diversity observed in the farming method, feeding and health monitoring in the different agroecological zones. The results will contribute to set a program improving the breeding systems.

Keywords: Breeding; Muscovy duck; Agro-ecological zone; Benin.

\section{INTRODUCTION}

L'aviculture familiale constitue une source non négligeable de protéines pour les paysans et les populations urbaines du Bénin. Elle concerne les poulets, les pintades, les canards, les dindons et les oies (DE, 2016). Parmi ces espèces, les poulets locaux couvrent $80 \%$ du cheptel avicole (DE, 2016) et permettent aux éleveurs de couvrir une partie des besoins nutritionnels de la famille. Ils sont également utilisés comme source d'épargne par leur vente (Youssao et al., 2013). Après les poulets locaux, la pintade locale est l'espèce la plus élevée en aviculture familiale au Bénin et de nombreux travaux ont été réalisés pour son développement, notamment sur sa caractérisation phénotypique pour l'exploitation de sa diversité (Houndonougbo, 2017), sur son système d'élevage (Chrysostome, 1995 ; Boko, 2012), sur l'amélioration de son alimentation (Dahouda, 2009), de ses performances de reproduction (Houndonougbo, 2017) et enfin sur la lutte contre les pathologies (Boko, 2012). Contrairement aux poulets locaux et à la pintade locale, le canard qui vient en troisième position après en terme numérique, est une espèce orpheline en raison d'une insuffisance de travaux scientifiques réalisés sur ces oiseaux et par sa marginalisation dans les Projets de développements. Or, le canard a une bonne 
performance de croissance que les poulets locaux et les pintades et résiste mieux que les poulets aux pathologies aviaires (Houessionon et Youssao, 2018). Son élevage est répandu sur tout le territoire national et particulièrement au Sud. L'espèce de canard la plus élevée au Bénin est Cairina moschata encore appelé canard de Barbarie. Un état des lieux de cet élevage est nécessaire pour diagnostiquer les goulots d'étranglement qui limitent le développement de

\section{MATERIELS ET METHODE}

Milieu d'étude: L'étude sur les caractéristiques de l'élevage des canards de Barbarie a été réalisée dans les exploitations des zones agro-écologiques du Sud Bénin : dépressions, pêcheries et terres de barre (figure 1).

Zone de dépression : La zone de dépression s'étend sur $2.564 \mathrm{~km}^{2}$ et est constituée des communes d'AdjaOuèrè, de Pobè, de Toffo, de Lalo et de Zogbodomey. La population était de 602843 en 2002 avec 391147 de ménages ruraux (PANA-BENIN, 2007). Le maïs associé au manioc, au niébé, à la tomate et au piment, constituent la base du système de production. C'est la plus petite des huit zones agro-écologiques du Bénin et elle est comparable à la zone de Terre de barre sur le plan climatique avec cependant une forte humidité relative d'environ $85 \%$.

Zone des pêcheries: La zone des pêcheries est la plus méridionale et occupe la zone fluvio-lacustre du Sud-Bénin. Elle couvre une superficie de $3.280 \mathrm{~km}^{2}$ et est composée des communes d'Athiémé, de GrandPopo, de Bopa, de Comé, de Lokossa, de Ouidah, de So-Ava, de Sèmè-Podji, d'Aguégués, de Dangbo, d'Adjohoun, de Bonou, de Ouinhi et de Cotonou. La cette spéculation en vue de mettre en place une stratégie d'amélioration. L'objectif de l'étude est de faire un état des lieux de l'élevage des canards de Barbarie dans les trois zones agro-écologiques (dépression, des pêcheries et des terres de barre) du Sud-Bénin. De façon spécifique, il s'agira de caractériser les élevages dans ces trois zones et de faire des suggestions pour l'intensification et le développement de l'élevage de cette espèce au Sud-Bénin.

population était de 1.435 .888 en 2002 et le nombre de ménage de 65120 (PANA-BENIN, 2007). Leur activité principalement est la pêche, ensuite l'agriculture (maïs en tête de rotation, le manioc, le niébé et les cultures maraîchères). La très faible disponibilité des terres limite l'extension de l'agriculture.

Zone des terres de barre: La zone des terres de barre couvre une superficie de $6.391 \mathrm{~km}^{2}$ et regroupe les communes d'Abomey-Calavi, d'Allada, de Kpomassè, de Tori-Bossito, de Zè, de Djakotomé, de Dogbo, de Klouékanmey, de Houéyogbé, de Toviklin, d'Adjarra, d'Ifangni, de Missérété, d'Avrankou, de Porto-Novo, de Sakété, d'Abomey, d'Agbangnizoun, de Bohicon, de Covè, de Zakpota et de Zagnanado. La population totale de cette zone était de 1.960 .136 en 2002 et le nombre de ménages ruraux de 144715 (PANA-BENIN, 2007). L'activité économique majeure des populations vulnérables de cette zone est l'agriculture (maïs en tête de rotation, manioc, niébé et arachide sont les principales spéculations). Dans cette zone, le régime des pluies est souvent perturbé entraînant des changements dans les cycles de production annuelle. 


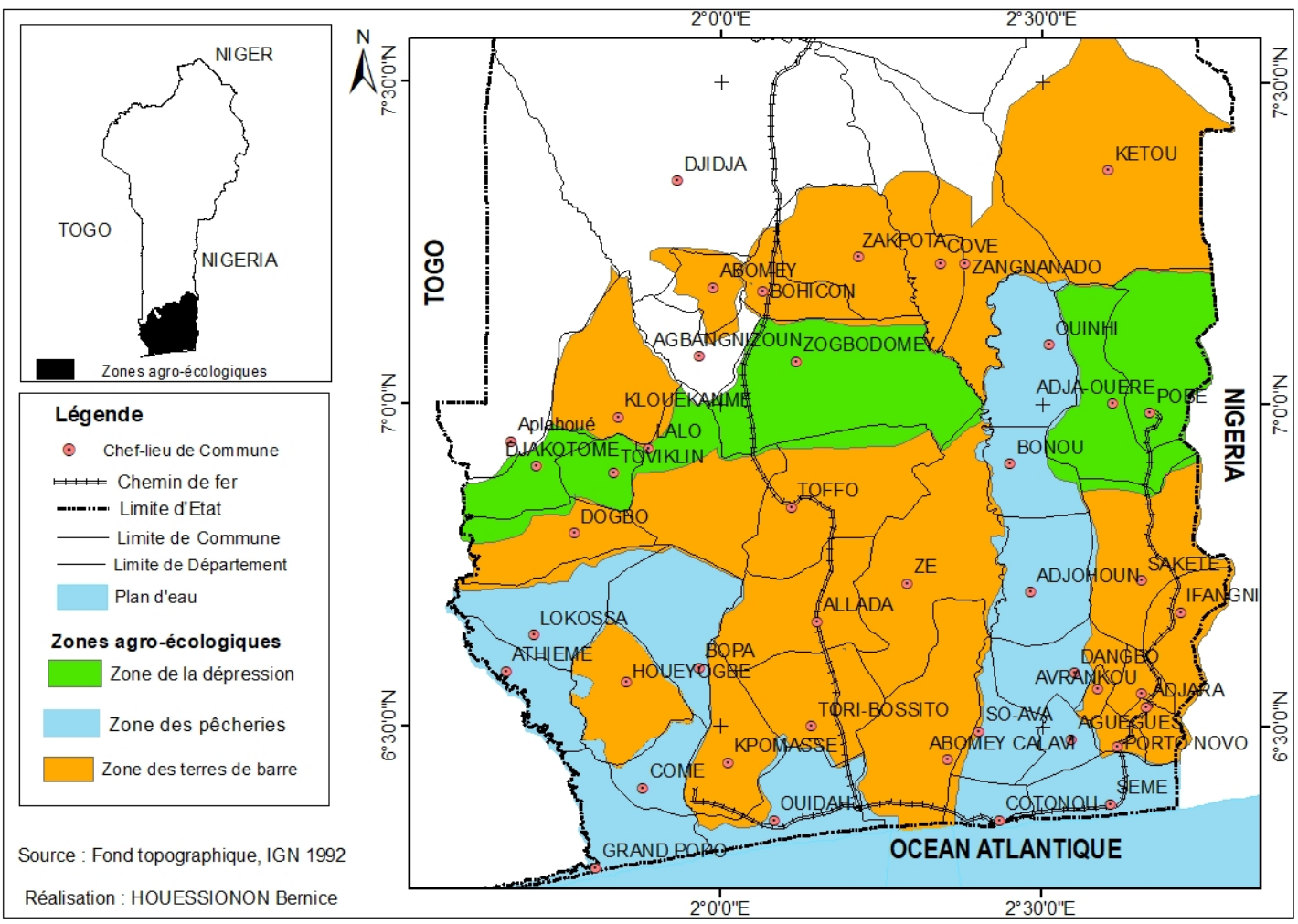

Figure 1: Carte du Bénin indiquant les zones agro-écologiques de l'étude

Matériel d'étude : Le matériel d'étude était constitué d'un guide d'entretien et des canards de Barbarie appelé Cairina moschata. Les questionnaires comportent les informations liées à l'identification de l'enquêté et du troupeau, à la conduite du troupeau, aux appréciations des caractéristiques de la carcasse et de la qualité de la viande selon les éleveurs enquêtés et au suivi sanitaire.

Méthode d'enquête : La méthodologie utilisée dans la présente étude a été celle de l'enquête rétrospective par entretien direct avec l'exploitant dans les communes des trois zones agro-écologiques. Elle fait appel à la mémoire des éleveurs, tout en reconstituant les différentes étapes d'élevage des canards de Barbarie. Dans chacune des communes, tous les éleveurs de canard barbarie ont été identifiés et le choix a été fait suivant des critères d'accessibilité et de disponibilité à fournir des informations. Chaque éleveur a été soumis à un entretien par discussion directe pour la collecte des données qui a été effective dans 213 élevages, de Juin 2015 à Août 2016.
Traitement des données : Après le dépouillement, les données ont été analysées avec le logiciel SAS (2013). La procédure Proc means a été utilisée pour la statistique descriptive. Pour les variables quantitatives (âge et la taille du cheptel), une analyse de variance à un seul facteur a été utilisée et la zone agro-écologique a été la seule source de variation. La procédure Proc GLM a été utilisée pour l'analyse de variance et le test de $F$ précise la signification de l'effet zone sur les variables considérées. Les comparaisons entre les moyennes ont été faites deux à deux par le test de $t$. Les fréquences ont été calculées par la procédure Proc freq du SAS (2013) et par le test bilatéral de Z. Pour chaque fréquence relative, un intervalle de confiance (IC) à $95 \%$ a été calculé suivant la formule :

$I C P=1,96 \sqrt{\frac{[P(1-P)]}{N}}$

où $\mathrm{P}$ est la fréquence relative et $\mathrm{N}$ la taille de l'échantillon. 


\section{RESULTATS}

Profil des éleveurs : L'âge moyen des éleveurs n'a pas varié d'une zone agro écologique à une autre $(p>0,05)$. II a été de 39 ans dans la zone de dépression, de 42 ans et de 41 ans respectivement dans les zones des pêcheries et des terres de barre. Dans les zones agro-écologiques des pêcheries et des terres de barre, 2 éleveurs sur 3 sont des hommes contre $45,45 \%$ dans la zone de dépression $(p<0,05)$. La proportion des femmes éleveurs dans la zone de dépression $(59,55 \%)$ est plus élevée $(p<0,05)$ que celles observées dans les zones des pêcheries et des terres de barre, qui sont respectivement de $32,14 \%$ et de $21,5 \%$. En outre, tous les éleveurs sont mariés et les pourcentages ne diffèrent pas d'une zone à une autre $(p<0,05)$. Plus de la moitié des ménages ont entre 1 et 5 enfants aves des proportions qui n'ont pas varié d'une zone à une autre : $72,73 \%, 61,82 \%$ et $58,59 \%$, respectivement pour la zone de dépression, la zone des terres de barre et la zone des pêcheries. Par rapport au niveau d'instruction, aucune différence n'a été observée entre les fréquences des éleveurs non scolarisés et ceux qui ont le niveau primaire dans les trois zones agro écologiques ( $p>0,05)$. Par contre, les zones des pêcheries $(22,78 \%)$ et des terres de barre $(32,69 \%)$ ont les proportions les plus élevées d'enquêtés ayant le niveau secondaire contre 4,55\% dans la zone de dépression $(p<0,05)$. Les éleveurs qui ont le niveau d'études supérieures sont plus rencontrés $(p<0,05)$ dans la zone de dépression $(13,64 \%)$ et dans la zone des terres de barre $(15,38 \%)$ que dans la zone des pêcheries $(3,8 \%)$. Les éleveurs pratiquent des activités très diverses, ils sont des ménagères, des agriculteurs, des éleveurs, des pêcheurs, des artisans, des salariés et des commerçants. Par ailleurs, aucun enquêté n'était éleveur professionnel dans la zone de dépression. De plus, la proportion des éleveurs professionnels enregistrée dans la zone des pêcheries $(14,46 \%)$ est supérieure $(p<0,05)$ à celle de la zone des terres de barre $(5,61 \%)$. Aussi, la fréquence des commerçants éleveurs dans les zones de dépression $(18,18 \%)$ est-elle plus élevée que celles de la zone des pêcheries $(7,23 \%)$ et de la zone des terres de barre $(5,61 \%)(p<0,05)$.

Importance sociale, culturelle, cultuelle et économique: Les éleveurs enquêtés dans les trois zones agro-écologiques pratiquent l'élevage des canards de Barbarie pour diverses raisons. Sur le plan social, $86,36 \%$ font cet élevage sans aucun motif précis dans la zone de dépression et cette proportion est significativement plus élevée $(p<0,05)$ que celles obtenues dans les zones des pêcheries $(59,04 \%)$ et des terres de barre $(53,27 \%)$. Les éleveurs de canards pratiquent cet élevage, plus pour le prestige dans les zones des pêcheries $(40,96 \%)$ et des terres de barre $(38,32 \%)$ que dans la zone de dépression $(1,42 \%)$. Les canards sont plus utilisés pour des sacrifices dans la zone de dépression $(36,36 \%)$ et dans la zone des pêcheries $(21,95 \%)$ que dans la zone des terres de barre $(16,98 \%)$. Par contre, les enquêtés qui élèvent les canards pour leur protection sont moins fréquents $(p<0,05)$ dans la zone de dépression $(4,55 \%)$ que dans les zones des pêcheries (36,59 \%) et de des terres de barre $(20,75 \%)$. Aucun éleveur dans les zones de dépression et des pêcheries n'utilise le canard pour la dot mais $14,15 \%$ de ces éleveurs le font dans la zone des terres de barre. L'élevage du canard est rentable chez $50 \%$ des éleveurs de la zone de dépression, 72,5 $\%$ des éleveurs des pêcheries et $17,92 \%$ des éleveurs des terres de barre.

Comparaison des performances d'élevage entre le canard de Barbarie et le poulet: L'habitat, l'alimentation, la rentabilité, la croissance et la rusticité des canards ont été comparés avec ceux du poulet (tableau 1a). Les canards sont moins exigeants que les poulets $(p<0,05)$ pour l'habitat dans les trois zones agro-écologiques. En matière d'alimentation, à l'exception des éleveurs de la zone des pêcheries qui rapportent que le canard est peu exigeant $(p<0,05)$ que le poulet, tous les autres n'observent pas de différences entre ces deux espèces. Le canard est plus rentable et plus rustique avec de bonnes performances de croissance que le poulet $(p<0,05)$ dans chacune des zones agro-écologiques. 
Tableau 1a : Comparaison des performances d'élevage entre le canard de Barbarie et le poulet

\begin{tabular}{|c|c|c|c|c|c|c|c|c|c|c|c|c|c|c|c|c|c|c|c|}
\hline \multirow{3}{*}{ Variables } & & \multicolumn{6}{|c|}{ Zone de dépression } & \multicolumn{6}{|c|}{ Zone des pêcheries } & \multicolumn{6}{|c|}{ Zone des terres de barre } \\
\hline & & \multicolumn{3}{|c|}{ Canard } & \multicolumn{3}{|c|}{ Poulet } & \multicolumn{3}{|c|}{ Canard } & \multicolumn{3}{|c|}{ Poulet } & \multicolumn{3}{|c|}{ Canard } & \multicolumn{3}{|c|}{ Poulet } \\
\hline & & $\mathbf{N}$ & $\%$ & IC & $\mathbf{N}$ & $\%$ & IC & $\mathrm{N}$ & $\%$ & IC & $\mathrm{N}$ & $\%$ & IC & $\mathbf{N}$ & $\%$ & IC & $\mathbf{N}$ & $\%$ & IC \\
\hline \multirow{4}{*}{ Habitat } & Pas exigeant & 22 & Oa & 0 & 20 & Oa & 0 & 84 & $8,32 a$ & 5,91 & 64 & $4,69 a$ & 5,18 & 106 & $18,87 a$ & 7,45 & 57 & $1,75 b$ & 3,4 \\
\hline & Peu exigeant & 22 & $86,36 a$ & 14,34 & 20 & $15 b$ & 15,34 & 84 & $58,33 a$ & 10,54 & 64 & $32,14 b$ & 11,5 & 106 & $38,68 a$ & 9,27 & 57 & $22,81 b$ & 10,89 \\
\hline & Exigeant & 22 & $13,64 b$ & 14,34 & 20 & $85 a$ & 15,34 & 84 & $32,14 b$ & 9,99 & 64 & $57,81 a$ & 12,1 & 106 & $42,45 b$ & 9,41 & 57 & $73,68 a$ & 11,43 \\
\hline & Très exigeant & 22 & $\mathrm{Oa}$ & 0 & 20 & $\mathrm{Oa}$ & 0 & 84 & $1,19 a$ & 2,32 & 64 & $4,69 a$ & 5,18 & 106 & $0 \mathrm{a}$ & 0 & 57 & $1,75 a$ & 3,4 \\
\hline \multirow{4}{*}{ Alimentation } & Pas exigeant & 22 & - & - & 16 & - & - & 84 & $1,19 a$ & 2,32 & 60 & $0 a$ & 0 & 105 & $7,62 a$ & 5,07 & 59 & ob & 0 \\
\hline & Peu exigeant & 22 & $0 \mathrm{a}$ & 0 & 16 & $6,25 a$ & 11,86 & 84 & $10,71 b$ & 6,13 & 60 & $26,67 a$ & 11,19 & 105 & $32,38 a$ & 8,95 & 59 & $37,29 a$ & 12,34 \\
\hline & Exigeant & 22 & $100 a$ & 12,54 & 16 & $93,75 a$ & 11,86 & 84 & $80,95 a$ & 8,4 & 60 & $70 a$ & 11,6 & 105 & $46,67 a$ & 9,54 & 59 & $57,63 a$ & 12,61 \\
\hline & Très exigent & 22 & $\mathrm{Oa}$ & 0 & 16 & $0 \mathrm{a}$ & 0 & 84 & $7,14 a$ & 5,51 & 60 & $3,33 a$ & 4,54 & 105 & $13,32 a$ & 6,5 & 59 & $5,08 a$ & 5,6 \\
\hline \multirow{4}{*}{ Rentabilité } & Pas rentable & 22 & $0 \mathrm{a}$ & 0 & 15 & $0 \mathrm{a}$ & 0 & 83 & $4,82 a$ & 4,61 & 56 & $5,36 a$ & 5,9 & 107 & $2,8 a$ & 3,13 & 56 & $0 a$ & 0 \\
\hline & Peu rentable & 22 & $18,18 b$ & 16,12 & 15 & $73,33 a$ & 22,32 & 83 & $18,07 \mathrm{~b}$ & 8,28 & 56 & $64,29 a$ & 12,55 & 107 & $8,41 b$ & 5,26 & 56 & $46,43 a$ & 13,06 \\
\hline & Rentable & 22 & $77,27 a$ & 17,51 & 15 & $26,67 \mathrm{~b}$ & 22,32 & 83 & $67,47 a$ & 10,08 & 56 & $28,57 \mathrm{~b}$ & 11,83 & 107 & $65,42 a$ & 9,01 & 56 & $48,21 b$ & 13,09 \\
\hline & Très rentable & 22 & $4,55 a$ & 8,71 & 15 & $0 \mathrm{a}$ & 0 & 83 & $9,64 a$ & 6,35 & 56 & $1,79 a$ & 3,47 & 107 & $23,36 a$ & 8,02 & 56 & $5,36 \mathrm{~b}$ & 5,9 \\
\hline \multirow{4}{*}{ Croissance } & Faible & - & - & - & 13 & - & - & - & - & - & - & - & - & - & - & - & - & - & - \\
\hline & Satisfaisant & 21 & $0 \mathrm{~b}$ & 0 & 13 & $84,62 a$ & 19,61 & 83 & $3,61 b$ & 4,13 & 56 & $78,57 a$ & 10,74 & 102 & $3,92 a$ & 3,77 & 52 & $71,15 a$ & 12,31 \\
\hline & Bonne & 21 & $90,48 a$ & 12,55 & 13 & $15,38 b$ & 19,61 & 83 & $77,11 a$ & 9,04 & 56 & $21,43 b$ & 10,74 & 102 & $62,75 a$ & 9,38 & 52 & $28,85 b$ & 12,31 \\
\hline & Très bonne & 21 & $9,52 \mathrm{a}$ & 12,55 & - & $0 \mathrm{a}$ & 0 & 83 & $19,28 a$ & 8,49 & 56 & $0 \mathrm{~b}$ & 0 & 102 & $33,33 a$ & 9,15 & 52 & $0 \mathrm{~b}$ & 0 \\
\hline \multirow{4}{*}{ Rusticité } & Pas rustique & 22 & $0 \mathrm{a}$ & 0 & 15 & $0 a$ & 0 & 83 & $0 \mathrm{a}$ & 0 & 58 & $0 \mathrm{a}$ & 0 & 107 & $28,04 a$ & 8,51 & 57 & ob & 0 \\
\hline & Peu rustique & 22 & $9,09 b$ & 12,01 & 15 & $80 a$ & 22,24 & 83 & $4,82 b$ & 4,61 & 58 & $74,14 a$ & 11,27 & 107 & Ob & 0 & 57 & $68,42 a$ & 12,07 \\
\hline & Rustique & 22 & $81,82 a$ & 16,12 & 15 & $20 \mathrm{~b}$ & 20,24 & 83 & $69,88 a$ & 9,87 & 58 & $22,41 b$ & 10,73 & 107 & $47,66 \mathrm{a}$ & 9,46 & 57 & $26,32 b$ & 11,43 \\
\hline & Très rustique & 22 & $9,09 \mathrm{a}$ & 12,01 & 15 & $0 \mathrm{a}$ & 0 & 83 & $25,3 a$ & 9,35 & 58 & $3,45 b$ & 4,7 & 107 & $24,3 a$ & 8,13 & 57 & $5,26 \mathrm{~b}$ & 5,8 \\
\hline
\end{tabular}

$\%$ : Fréquence, IC: Intervalle de confiance. Les pourcentages de la même ligne suivis des lettres différentes, diffèrent significativement au seuil de $5 \%$; $\mathrm{N}$ : effectif total

Tableau 1b: L'âge à l'abattage et nombre d'œufs pondus du canard avec ceux du poulet

\begin{tabular}{|c|c|c|c|c|c|c|c|}
\hline \multirow{2}{*}{ Variables } & \multicolumn{2}{|c|}{ Zone de dépression } & \multicolumn{2}{|c|}{ Zone des Pêcherie } & \multicolumn{2}{|c|}{ Zone des terres de barre } & \multirow{2}{*}{ ANOVA } \\
\hline & Moyenne & ES & Moyenne & ES & Moyenne & ES & \\
\hline Age à l'abattage du canard (mois) & $7,1 \mathrm{a}$ & 0,86 & $7,19 a$ & 0,31 & $7,19 a$ & 0,27 & NS \\
\hline Age à l'abattage du poulet (mois) & $5,85 a b$ & 0,24 & $5,38 a$ & 0,22 & $6,09 b$ & 0,25 & * \\
\hline Nombre d'œuf par la cane & $9,48 a$ & 1,13 & $12,46 b$ & 0,57 & $10,69 a$ & 0,51 & * \\
\hline Nombre d'œuf par poule & $8,98 \mathrm{a}$ & 0,67 & $8,78 \mathrm{a}$ & 0,57 & $9,07 a$ & 0,71 & NS \\
\hline
\end{tabular}

ES : Erreur Standard ; moyenne suivies des lettres différentes diffèrent significativement au seuil de $5 \% ; N S: p>0,05,{ }^{*}: p<0,05$ 
Age à l'abattage et nombre d'œufs pondus : L'âge à l'abattage et la productivité du canard et du poulet selon les éleveurs des trois zones sont présentés dans le tableau $1 \mathrm{~b}$. L'âge moyen à l'abattage des canards est de 7 mois et ne varie pas d'une zone agro écologique du Sud-Bénin à une autre $(p>0,05)$. L'âge à l'abattage des poulets locaux de la zone agro écologique des terres de barre (6 mois) est significativement plus élevé que celui de la zone agro écologique des pêcheries (5,38 mois), alors que celui de la zone de dépression (5,85 mois) ne diffère pas de ceux des autres zones. Chez la cane, le nombre d'œufs le plus élevé $(p<0,05)$ a été rapporté dans la zone des pêcheries (12,46 œufs) et les plus faibles sont rapportés dans les zones des terres de barre $(10,69$ œufs) et de dépression (9,48 œufs). Chez la poule, le nombre d'œufs pondus ne varie pas d'une zone agro écologique à une autre.
Comparaison de la qualité de la carcasse et de la viande du canard de Barbarie à celle du poulet : Les avis des enquêtés ont été très diversifiés en ce qui concerne la carcasse des canards de Barbarie et des poulets (tableau 2). En dehors des enquêtés de la zone des terres de barre chez qui la qualité de la carcasse du canard est bonne par rapport à celle des poulets (66,98 vs $54 \%$ ), au moins $50 \%$ des enquêtés rapportent que la carcasse des canards a une qualité bonne autant que celle des poulets. Toutefois, moins de $45 \%$ des enquêtés rapportent, dans chacune des trois zones agro-écologiques, que la qualité de la carcasse du canard est très bonne par rapport à celle du poulet $(p<0,05)$. L'abdomen est plus gras chez le canard que chez le poulet $(p<0,05)$, il en est de même pour la carcasse. 
Tableau 2: Qualités de la carcasse et de la viande du canard de Barbarie comparées à celles du poulet

\begin{tabular}{|c|c|c|c|c|c|c|c|c|c|c|c|c|c|c|c|c|c|c|c|}
\hline \multirow[t]{3}{*}{ Variables } & & \multicolumn{6}{|c|}{ Zone de dépression } & \multicolumn{6}{|c|}{ Zone des pêcheries } & \multicolumn{6}{|c|}{ Zone des terres de barre } \\
\hline & & \multicolumn{3}{|c|}{ Canard } & \multicolumn{3}{|c|}{ Poulet } & \multicolumn{3}{|c|}{ Canard } & \multicolumn{3}{|c|}{ Poulet } & \multicolumn{3}{|c|}{ Canard } & \multicolumn{3}{|c|}{ Poulet } \\
\hline & & $\mathbf{N}$ & $\%$ & IC & $\mathbf{N}$ & $\%$ & IC & $\mathbf{N}$ & $\%$ & IC & $\mathbf{N}$ & $\%$ & IC & $\mathbf{N}$ & $\%$ & IC & $\mathbf{N}$ & $\%$ & IC \\
\hline Poids & Médiocre & 18 & $0 \mathrm{a}$ & 0 & 18 & $0 \mathrm{a}$ & 0 & 82 & $2,44 a$ & 3,34 & 60 & $0 \mathrm{a}$ & 0 & 106 & $0 \mathrm{a}$ & 0 & 54 & $0 \mathrm{a}$ & 0 \\
\hline \multirow[t]{3}{*}{ carcasse } & Passable & 18 & $5,56 b$ & 10,59 & 18 & $33,33 a$ & 21,78 & 82 & $4,88 b$ & 4,66 & 60 & $41,33 a$ & 12,46 & 106 & $1,89 b$ & 2,59 & 54 & $53,7 a$ & 13,3 \\
\hline & Bonne & 18 & $50 a$ & 23,1 & 18 & $56,56 a$ & 22,9 & 82 & $51,22 a$ & 10,82 & 60 & $55 a$ & 12,59 & 106 & $66,98 a$ & 8,95 & 54 & $42,59 b$ & 13,19 \\
\hline & $\begin{array}{l}\text { Très } \\
\text { bonne }\end{array}$ & 18 & $44,44 a$ & 22,96 & 18 & $11,11 \mathrm{~b}$ & 14,52 & 82 & $41,46 \mathrm{a}$ & 10,66 & 60 & $3,3 b$ & 4,52 & 106 & $31,13 a$ & 8,81 & 54 & $3,7 \mathrm{~b}$ & 5,03 \\
\hline \multirow[t]{4}{*}{ Abdomen } & Maigre & 18 & $0 \mathrm{a}$ & 0 & 16 & $0 \mathrm{a}$ & 0 & 79 & $7,59 a$ & 5,84 & 60 & $0 \mathrm{a}$ & 0 & 106 & $\mathrm{Ob}$ & 0 & 55 & $14,55 \mathrm{a}$ & 9,32 \\
\hline & Peu gras & 18 & $5,56 \mathrm{~b}$ & 10,59 & 16 & $56,25 a$ & 24,31 & 79 & $15,19 b$ & 7,91 & 60 & $80 a$ & 10,12 & 106 & $6,6 \mathrm{~b}$ & 4,73 & 55 & $74,55 a$ & 11,51 \\
\hline & Gras & 18 & $88,89 a$ & 14,52 & 16 & $43,75 b$ & 24,31 & 79 & $72,15 a$ & 9,88 & 60 & $20 \mathrm{~b}$ & 10,12 & 106 & $58,49 a$ & 9,38 & 55 & $10,91 b$ & 8,24 \\
\hline & Très gras & 18 & $5,56 a$ & 10,59 & 16 & $0 \mathrm{a}$ & 0 & 79 & $5,06 a$ & 4,83 & 60 & $0 \mathrm{a}$ & 0 & 106 & $34,91 \mathrm{a}$ & 9,07 & 55 & $0 \mathrm{~b}$ & 0 \\
\hline \multirow[t]{4}{*}{ Carcasse } & Gras & 19 & $100 a$ & 0 & 10 & $30 \mathrm{~b}$ & 28,4 & 80 & $56,25 a$ & 10,87 & 58 & $27,79 b$ & 11,53 & 105 & $36,19 a$ & 9,19 & 54 & $5,56 \mathrm{~b}$ & 6,11 \\
\hline & $\begin{array}{l}\text { Peu } \\
\text { maigre }\end{array}$ & 19 & $0 \mathrm{~b}$ & 0 & 10 & $60 a$ & 30,36 & 80 & $35 b$ & 10,45 & 58 & $55,17 a$ & 12,8 & 105 & $41,9 \mathrm{a}$ & 9,44 & 54 & $51,85 a$ & 13,33 \\
\hline & Maigre & 19 & $0 \mathrm{a}$ & 0 & 10 & $10 \mathrm{~b}$ & 18,59 & 80 & $7,5 b$ & 5,77 & 58 & $17,24 a$ & 9,72 & 105 & $19,05 b$ & 7,51 & 54 & $40,74 a$ & 13,11 \\
\hline & $\begin{array}{l}\text { Très } \\
\text { maigre }\end{array}$ & 19 & $0 \mathrm{a}$ & 0 & 10 & $0 \mathrm{a}$ & 0 & 80 & $1,25 a$ & 2,43 & 58 & $0 \mathrm{a}$ & 0 & 105 & $2,86 a$ & 3,19 & 54 & $1,85 a$ & 3,59 \\
\hline
\end{tabular}

$\%$ : Fréquence, IC : Intervalle de confiance, les pourcentages de la même ligne suivis des lettres différentes diffèrent significativement au seuil de $5 \%$; $\mathrm{N}$ : effectif total

Tableau 3: Historique de l'exploitation et motivation vis-à-vis de l'élevage de canard

\begin{tabular}{|c|c|c|c|c|c|c|c|c|c|c|c|}
\hline \multirow{2}{*}{ Variables } & & & \multicolumn{3}{|c|}{ Zone de dépression } & \multicolumn{3}{|c|}{ Zone des pêcheries } & \multicolumn{3}{|c|}{ Zone des terres de barre } \\
\hline & & & $\mathbf{N}$ & $\%$ & IC & $\mathbf{N}$ & $\%$ & IC & $\mathbf{N}$ & $\%$ & IC \\
\hline \multirow{3}{*}{$\begin{array}{l}\text { Date de création des } \\
\text { exploitations }\end{array}$} & 0 à 1987 & & 22 & $0 \mathrm{a}$ & 0 & 83 & $4,8 a$ & 4,6 & 105 & $9,5 a$ & 5,61 \\
\hline & 1990 à 1999 & & 22 & $\mathrm{Ob}$ & 0 & 83 & $12,03 b$ & 7 & 105 & $26,65 a$ & 8,46 \\
\hline & 2000 à 2016 & & 22 & $100 \mathrm{a}$ & 0 & 83 & $83,15 b$ & 8,05 & 105 & $62,84 \mathrm{c}$ & 9,24 \\
\hline \multirow{2}{*}{\multicolumn{2}{|c|}{$\begin{array}{l}\text { Provenance des animaux lors de la } \\
\text { création }\end{array}$}} & Achat & 22 & $95,45 a$ & 8,71 & 84 & $96,43 a$ & 3,97 & 107 & $96,26 a$ & 3,6 \\
\hline & & Don & 22 & $4,55 a$ & 8,71 & 84 & $3,57 a$ & 3,97 & 107 & $6,64 a$ & 4,718 \\
\hline \multirow{3}{*}{\multicolumn{2}{|c|}{ Type de Production }} & Viande & 22 & $100 a$ & 0 & 84 & $100 a$ & 0 & 107 & $98,13 a$ & 2,57 \\
\hline & & OEufs & 22 & $0 a$ & 0 & 84 & $11,9 a$ & 6,92 & 107 & $1,87 b$ & 2,57 \\
\hline & & Culture & 22 & $0 \mathrm{a}$ & 0 & 84 & $14,29 a$ & 7,48 & 107 & $4,67 \mathrm{~b}$ & 4 \\
\hline \multirow{3}{*}{\multicolumn{2}{|c|}{$\begin{array}{l}\text { Motivation vis-à-vis de l'élevage du canard } \\
\text { barbarie }\end{array}$}} & Facilité & 22 & $100 a$ & 0 & 79 & $74,68 b$ & 9,59 & 107 & $95,33 a$ & 4 \\
\hline & & Rusticité & 22 & $O b$ & 0 & 79 & $16,83 a$ & 8,25 & 107 & $16,02 a$ & 6,95 \\
\hline & & Tradition & 22 & $0 a$ & 0 & 79 & $15,19 a$ & 7,91 & 107 & $11,21 a$ & 5,98 \\
\hline
\end{tabular}

$\%$ : Fréquence ; IC : Intervalle de confiance, les pourcentages de la même ligne suivis des lettres différentes diffèrent significativement au seuil de $5 \%$; $\mathrm{N}$ : effectif total 
Avantages et inconvénients de l'élevage du canard de Barbarie : Les enquêtés font l'élevage du canard de Barbarie pour plusieurs raisons. En effet, 47,06\% des éleveurs de la zone de dépression, $26,51 \%$ de la zone des pêcheries et $29,13 \%$ de la zone des terres de barre élèvent les canards de Barbarie à cause de la prolificité des canes. Les fréquences des aviculteurs qui élèvent les canards de Barbarie à cause de la rentabilité dans la zone des pêcheries $(40,96 \%)$ et dans la zone des terres de barre $(44,66 \%)$ sont plus élevés $(p<0,05)$ que celle obtenue dans la zone de dépression $(11,76 \%)$. Les proportions des éleveurs qui font cet élevage pour se protéger spirituellement et la rusticité des oiseaux dans les trois zones sont identiques. C'est seulement dans la zone de dépression qu'une minorité $(11,76 \%)$ des enquêtés pratique cet élevage à cause de la bonne performance de croissance des canetons. Plusieurs raisons limitent l'essor de la production de ces oiseaux dans le SudBénin. Ainsi, 27,27 \%, 16,05 \% et 22,86 \% des éleveurs, respectivement dans la zone de dépression, la zone des pêcheries et la zone des terres de barre ont rapporté que l'inexistence du marché d'écoulement constitue un frein pour cet élevage. Le taux élevé de mortalité des canetons, la pollution de l'environnement par les déjections et l'aspect sacré de l'espèce, sont autant de facteurs qui limitent le développement de cette spéculation chez certains (moins de $15 \%$ ) éleveurs enquêtés.

Types d'exploitation: Dans les élevages enquêtés, en dehors des canards de Barbarie, d'autres espèces sont présentes. Les poulets sont plus élevés $(p<0,05)$ dans les exploitations de la zone de dépression $(95 \%)$ que dans celles des zones des pêcheries $(73,81 \%)$ et des terres de barre $(73,83 \%)$. Les pintades sont élevées dans $14,29 \%$ et $14,95 \%$ des exploitations enquêtées, respectivement dans les zones des pêcheries et des terres de barre alors que les oies sont absentes dans presque toutes ces exploitations. D'autres espèces telles que les bovins, les ovins, les caprins, les porcs et les lapins sont signalées dans les exploitations enquêtées. Les canards sont dominants dans 1 exploitation sur 3 dans les zones de dépression et des terres de barre, et dans $43,37 \%$ des élevages de la zone des pêcheries $(p>0,05)$. Par contre, dans $52,38 \%$ des élevages de la zone de dépression, les poulets sont dominants et cette fréquence est plus élevée $(p<0,05)$ que celles enregistrées dans les zones des pêcheries $(15,66 \%)$ et des terres de barre $(37,38$ $\%$ ).

Historique des exploitations et motivation vis-à-vis de l'élevage de canard: Le tableau 3 présente l'historique des exploitations et la motivation des éleveurs vis-à-vis de l'élevage du canard de Barbarie. Tous les élevages de la zone de dépression ont été créés entre 2000 et 2016 , contre $83 \%$ dans la zone des pêcheries et $63 \%$ dans la zone des terres de barre. Les canards présents dans les élevages lors de la création de l'exploitation dans les trois zones agroécologiques sont achetés et sont élevés pour la viande. Tous les éleveurs de la zone de dépression (100\%) et la quasi-totalité de ceux des terres de barre $(95,33 \%)$, élèvent le canard à cause de la facilité de son élevage, contre 3 éleveurs sur $4 \quad(p<0,05)$ dans la zone des pêcheries $(74,68 \%)$.

Identification des oiseaux: Les méthodes d'identifications des oiseaux dans les différentes exploitations sont illustrées par la figure 2. Le moyen le plus courant qu'utilise la majorité des éleveurs des trois zones agro écologiques pour l'identification des canards est la couleur du plumage. La moitié des éleveurs l'utilise dans les zones de dépression et des pêcheries contre les deux tiers dans la zone des terres de barre $(p<0,05)$. Viennent ensuite les éleveurs de canard qui identifient les oiseaux par des caractéristiques phénotypiques visibles (aspects et couleurs de la caroncule, la forme et la couleur du bec, la couleur des tarses, etc.). La fréquence de ces éleveurs est plus élevée dans la zone des terres de barres que dans la zone des pêcheries $(p<0,05)$ pendant que la zone de dépression a présenté la plus faible fréquence $(p<0,05)$. En dehors de ces deux moyens d'identification des animaux, certains éleveurs utilisent des bagues, amputent un des doigts aux canards ou attachent des ficèles aux ailes des oiseaux. 


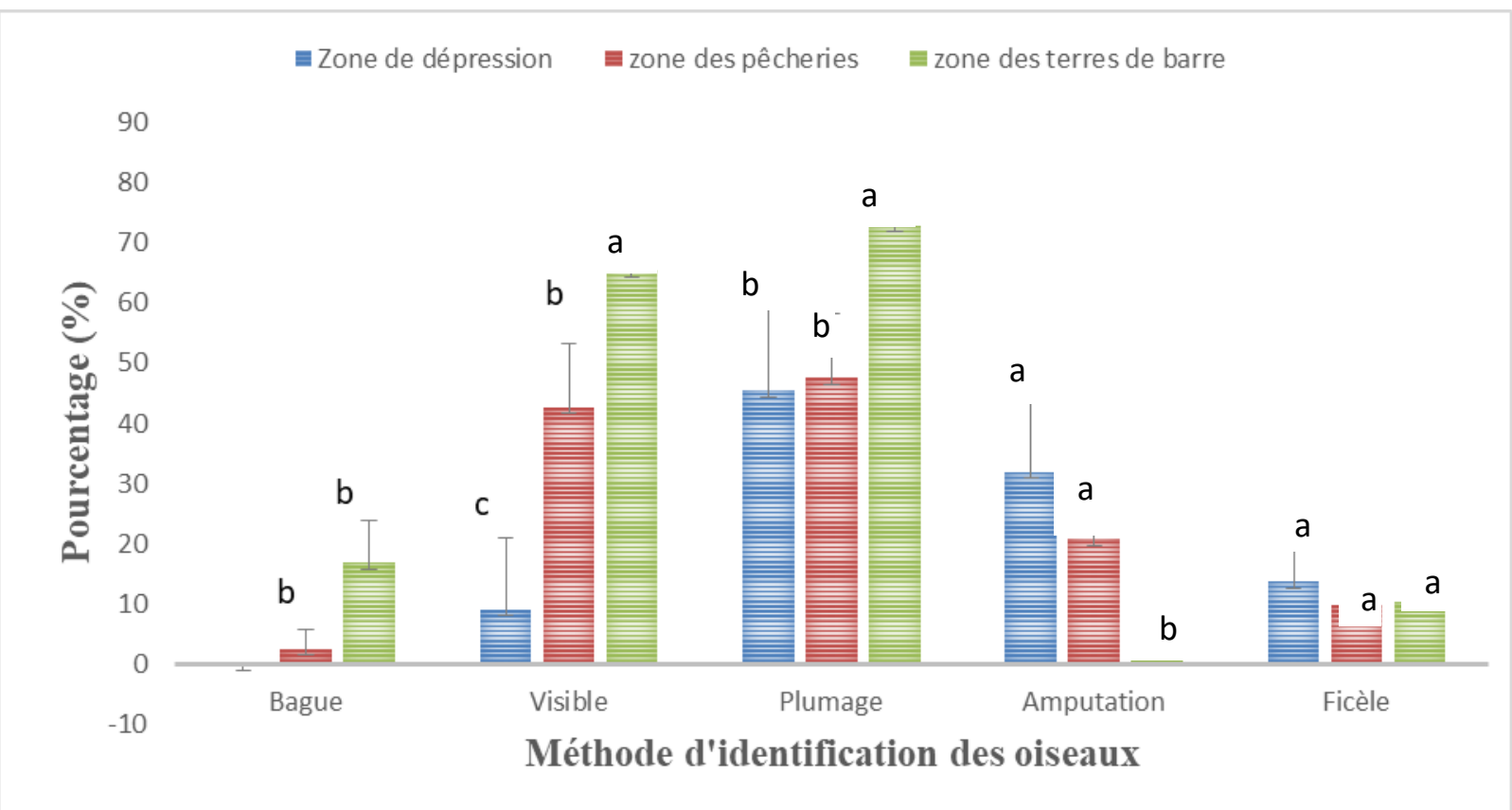

Figure 2 : Méthodes d'identification des canards de Barbarie par zone agro écologique

Structure des exploitations : Le tableau 4 présente la structure des exploitations de canard de Barbarie par zone agro-écologique. Le nombre total de canards de Barbarie par exploitation, le nombre de reproducteurs mâles et femelles, le nombre de jeunes canards, le nombre de canetons à l'éclosion, le nombre de ponte par saison et l'âge à l'entrée en reproduction des mâles et des femelles n'ont pas varié d'une zone agroécologique à une autre $(p>0,05)$. Par contre, le nombre de canetons sevrés dans la zone de dépression (7 canetons) et dans la zone des terres de barre (6 canetons) est inférieur $(p<0,01)$ à celui enregistré dans la zone des pêcheries (12 canetons). Par ailleurs, le nombre d'œufs pondus par cane et le nombre d'œufs incubés observé dans les élevages de la zone de dépression ne diffèrent pas de ceux des zones des pêcheries et des terres de barre $(p>0,05)$. Par contre, le nombre d'œufs pondus par cane et le nombre d'œufs incubés enregistrées dans la zone des pêcheries sont plus élevés $(p<0,05)$ à ceux de la zone des terres de barre. La mortalité a l'éclosion et au sevrage est plus élevée $(p<0,05)$ dans la zone des pêcheries que dans les deux autres zones. 
Tableau 4 : Structure des exploitations

\begin{tabular}{|c|c|c|c|c|c|c|c|}
\hline \multirow[t]{2}{*}{ Variables } & \multicolumn{2}{|c|}{ Zone de dépression } & \multicolumn{2}{|c|}{ Zone des pêcheries } & \multicolumn{2}{|c|}{ Zone des terres de barre } & \multirow[t]{2}{*}{ ANOVA } \\
\hline & Moyenne & ES & Moyenne & ES & Moyenne & ES & \\
\hline Nombre total d'animaux & $15,62 a$ & 6,81 & $29,93 a$ & 3,48 & $22,1 a$ & 3,09 & NS \\
\hline Nombre de mâles & $6,27 a$ & 2,9 & $11,83 a$ & 1,49 & $9,68 a$ & 1,31 & NS \\
\hline Nombre de femelles & $8,55 a$ & 4,10 & $17,37 a$ & 2,11 & $11,94 a$ & 1,86 & NS \\
\hline Nombre de canetons sevrés & $7,13 b$ & 2,58 & $12,06 a$ & 1,32 & $6,63 b$ & 1,18 & ** \\
\hline Nombre de jeunes canards & $3,86 a$ & 4,62 & $9,00 \mathrm{a}$ & 2,36 & $8,01 a$ & 2,11 & NS \\
\hline Nombre de reproducteurs mâles & $1,55 a$ & 0,63 & $3,14 a$ & 0,32 & $2,81 \mathrm{a}$ & 0,29 & NS \\
\hline Nombre de reproducteurs femelles & $3,36 a$ & 2,19 & $6,08 a$ & 1,13 & $6,53 a$ & 0,99 & NS \\
\hline Age à l'entrée en reproduction du mâle & $8,53 a$ & 0,52 & $8,03 a$ & 0,24 & $8,18 a$ & 0,21 & NS \\
\hline Age à l'entrée en reproduction de la femelle & $7,33 a$ & 0,46 & $6,93 a$ & 0,21 & $6,94 a$ & 0,18 & NS \\
\hline Nombre d'œufs pondus & $16,86 a b$ & 0,95 & $17,59 a$ & 0,49 & $15,93 b$ & 0,43 & * \\
\hline Nombre d'œufs incubés & $16,86 a b$ & 0,95 & $17,55 a$ & 0,49 & $15,93 b$ & 0,43 & * \\
\hline Nombre de canetons à l'éclosion & $13,36 a$ & 0,97 & $15,12 a$ & 0,50 & $13,71 a$ & 0,44 & NS \\
\hline Nombre de pontes par saison & $2,57 a$ & 0,21 & $2,59 a$ & 0,11 & $2,80 a$ & 0,09 & NS \\
\hline Mortalité à l'éclosion & $13,36 a$ & 0,96 & $15,18 \mathrm{a}$ & 0,49 & $13,62 b$ & 0,44 & * \\
\hline Mortalité au sevrage & $9,23 b$ & 1,10 & $12,35 a$ & 0,57 & $10,67 b$ & 0,5 & * \\
\hline
\end{tabular}

ES : Erreur Standard ; Moyennes suivies des lettres différentes diffèrent significativement au seuil de $5 \%$;

\section{Tableau 5 : Mode de reproduction et de production}

\begin{tabular}{|c|c|c|c|c|c|c|c|c|c|c|}
\hline \multirow{2}{*}{ Variables } & & \multicolumn{3}{|c|}{ Zone de dépression } & \multicolumn{3}{|c|}{ Zone des pêcheries } & \multicolumn{3}{|c|}{ Zone des terres de barre } \\
\hline & & $\mathbf{N}$ & $\%$ & IC & $\mathbf{N}$ & $\%$ & IC & $\mathbf{N}$ & $\%$ & IC \\
\hline \multirow{2}{*}{ Mode de reproduction } & Deux parents connus & 22 & $0 \mathrm{c}$ & 0 & 84 & $16,67 \mathrm{~b}$ & 7,97 & 107 & $46,73 a$ & 9,45 \\
\hline & Un parent connu & 22 & $100 a$ & 0 & 84 & $86,9 a$ & 7,22 & 107 & $54,21 b$ & 9,44 \\
\hline \multirow{2}{*}{ Moment de ponte } & Saisonnier & 21 & $100 a$ & 0 & 79 & $77,22 \mathrm{~b}$ & 9,25 & 104 & $95,19 a$ & 4,11 \\
\hline & Non saisonnier & 22 & $0 \mathrm{~b}$ & 0 & 84 & $30,38 \mathrm{a}$ & 9,84 & 107 & $11,54 \mathrm{~b}$ & 6,05 \\
\hline \multirow{2}{*}{$\begin{array}{l}\text { Période maximale de } \\
\text { ponte }\end{array}$} & Saison pluvieuse & 22 & $40,91 a$ & 20,55 & 84 & $39,29 a$ & 10,44 & 107 & $31,78 a$ & 8,82 \\
\hline & Saison sèche & 22 & $59,09 a$ & 20,55 & 84 & $60,71 \mathrm{a}$ & 10,44 & 107 & $68,22 a$ & 8,82 \\
\hline \multirow{5}{*}{$\begin{array}{l}\text { Critères de choix des } \\
\text { reproducteurs }\end{array}$} & Qualité recherchée & 22 & $0 c$ & 0 & 70 & $44,29 a$ & 11,64 & 102 & $19,61 b$ & 7,71 \\
\hline & Défaut à éliminer & 22 & $0 \mathrm{a}$ & 0 & 70 & $8,57 a$ & 6,56 & 102 & $4,9 a$ & 4,19 \\
\hline & Hasard & 22 & $100 a$ & 0 & 71 & $53,52 \mathrm{c}$ & 11,6 & 103 & $77,67 \mathrm{~b}$ & 8,04 \\
\hline & Bonne pondeuse et couveuse & - & - & - & 31 & $64,52 \mathrm{a}$ & 16,84 & 20 & $85 a$ & 15,65 \\
\hline & recherchées & - & - & - & 31 & $35,48 a$ & 16,84 & 20 & $15 a$ & 15,65 \\
\hline
\end{tabular}

$\%$ : Fréquence ; IC : Intervalle de confiance ; les pourcentages de la même ligne suivis des lettres différentes diffèrent significativement au seuil de $5 \%$; $\mathrm{N}$ : effectif total 
Mode de reproduction et de production : Le tableau 5 présente le mode de reproduction et de production du canard de Barbarie dans les trois zones agroécologiques du Sud-Bénin. Tous les éleveurs de la zone de dépression (100\%) et $86,9 \%$ de ceux de la zone des pêcheries, connaissent un des deux parents géniteurs des canards élevés dans leur exploitation. Ces deux fréquences des éleveurs enquêtés sont respectivement plus élevées $(p<0,05)$ que celle des exploitations de la zone des terres de barre $(54,21 \%)$. Dans toutes les exploitations des trois zones agroécologiques enquêtées, l'incubation des œufs se fait par la cane. La totalité des éleveurs de la zone de dépression et la quasi-totalité $(95,19 \%)$ de ceux de la zone des terres de barre rapportent que la ponte est saisonnière. Ces deux fréquences sont significativement plus élevées que celle de la zone des pêcheries $(P<0,05)$. La période maximale de ponte pour la majorité des éleveurs dans les trois zones agroécologiques est la saison sèche. De plus, tous les éleveurs de la zone de dépression et 3 éleveurs sur 4 dans la zone des terres de barre, choisissent les reproducteurs au hasard et près de la moitié des éleveurs de la zone des pêcheries tient compte des caractères phénotypiques (bonne aptitude à la croissance, bonne pondeuse et bonne couveuse).

Mode d'élevage et exploitation des canards de Barbarie : Le tableau 6 présente le mode d'élevage et l'utilisation des canards dans les trois zones agroécologiques du Sud du Bénin. La quasi-totalité des éleveurs de la zone de dépression $(90,91 \%)$ pratique l'élevage en divagation contre 52 et $64 \%$ des éleveurs, respectivement dans zones agro-écologiques des pêcheries et des terres de barre $(p<0,05)$. Toutefois, 1 éleveur sur 3 dans les zones des pêcheries et des terres de barre fait cet élevage en plein air ou offre un bâtiment plus un parcours aux canards. Les céréales et les sous-produits agricoles sont utilisés pour nourrir les oiseaux. La quasi-totalité des éleveurs des trois zones agro écologiques du Sud Bénin fait l'élevage des canards de Barbaries pour la commercialisation. Toutefois, les éleveurs des zones des pêcheries $(57,14$ $\%$ ) et ceux des terres de barre $(67,29 \%)$ consomment plus $(p<0,05)$ les canards qu'ils élèvent comparativement aux éleveurs de la zone de dépression $(31,82 \%)$. Les animaux sont souvent vendus lorsqu'ils ont atteint au moins l'âge à l'abattage chez tous les éleveurs enquêtés. Toutefois, ces animaux sont vendus lorsqu'ils deviennent vicieux ou sont en arrêt de ponte ou pour des raisons financières.

Mortalité et morbidité : Le tableau 7 présente la morbidité et la mortalité chez les canards de Barbarie dans les trois zones agro-écologiques du Sud-Bénin. Dans la plupart des élevages des trois zones (89 à 100 $\%)$, la mortalité chez les canards de Barbarie est plus remarquée avant le sevrage. Les causes de cette mortalité sont les pathologies, les écrasements par des véhicules, l'eau de ruissellement pendant la saison des pluies et la prédation. Le taux de mortalité le plus élevé est obtenu dans la zone de dépression $(86,3 \%)$ contre $53,16 \%$ et $39,62 \%$, respectivement dans les zones des pêcheries et des terres de barre $(p<0,05)$. La morbidité est plus remarquée chez les canetons avant le sevrage. Parmi les pathologies citées, la maladie de Marek est la plus rapportée par les éleveurs dans les zones de dépression et des pêcheries, tandis que la maladie de Newcastle est la plus citée dans la zone des terres de barre que dans les deux autres $(p<0,05)$. 
Tableau 6: Mode d'élevage et utilisation des oiseaux

\begin{tabular}{|c|c|c|c|c|c|c|c|c|c|c|}
\hline \multirow{2}{*}{ Variables } & & \multicolumn{3}{|c|}{ Zone de dépression } & \multicolumn{3}{|c|}{ Zone des pêcheries } & \multicolumn{3}{|c|}{ Zone des terres de barre } \\
\hline & & $\mathrm{N}$ & $\%$ & IC & $\mathrm{N}$ & $\%$ & IC & $\mathbf{N}$ & $\%$ & IC \\
\hline \multirow{4}{*}{ Mode d'élevage } & Bâtiment permanant & 22 & $0 \mathrm{a}$ & 0 & 84 & $4,76 a$ & 4,55 & 107 & $0,93 a$ & 1,82 \\
\hline & Bâtiment plus parcours & 22 & $\mathrm{Ob}$ & 0 & 84 & $27,38 a$ & 9,54 & 107 & $28,04 a$ & 8,51 \\
\hline & Plein air & 22 & $9,09 \mathrm{~b}$ & 12,01 & 84 & $32,14 a$ & 9,99 & 107 & $28,97 a$ & 8,6 \\
\hline & Divagation & 22 & $90,91 a$ & 12,01 & 84 & $52,38 b$ & 10,68 & 107 & $63,55 \mathrm{~b}$ & 9,12 \\
\hline \multirow{4}{*}{ Alimentation } & Céréales et sous-produits & 22 & $100 a$ & 0 & 84 & $95,24 a$ & 4,55 & 107 & $69,16 b$ & 8,75 \\
\hline & Provende & 22 & $31,82 b$ & 19,46 & 84 & $66,67 a$ & 10,08 & 107 & $71,96 a$ & 8,51 \\
\hline & Reste de cuisine & 22 & $100 a$ & 0 & 84 & $70,24 b$ & 9,78 & 107 & $82,24 b$ & 7,24 \\
\hline & Autres & 21 & $\mathrm{Ob}$ & 0 & 78 & $42,31 \mathrm{a}$ & 10,96 & 107 & $28,97 a$ & 8,6 \\
\hline \multirow{3}{*}{ Utilisation des oiseaux } & Auto consommation & 22 & $31,82 b$ & 19,46 & 84 & $57,14 a$ & 10,58 & 107 & $67,29 a$ & 8,89 \\
\hline & Vente & 22 & $95,45 a$ & 8,71 & 84 & $95,24 a$ & 4,55 & 107 & $97,2 \mathrm{a}$ & 3,13 \\
\hline & Autre & 21 & $\mathrm{Ob}$ & 0 & 78 & $42,31 \mathrm{a}$ & 10,96 & 107 & $28,97 a$ & 8,6 \\
\hline \multirow{6}{*}{ Moment de vente des oiseaux } & En âge de vente & 21 & 100 & 0 & 79 & 100 & 0 & 107 & 100 & 0 \\
\hline & Pendant la rentrée & 21 & $0 b$ & 0 & 79 & $2,53 b$ & 3,46 & 107 & $12,15 a$ & 6,19 \\
\hline & En cas de maladie & 21 & Oa & 0 & 79 & $1,27 a$ & 2,47 & 107 & $1,87 a$ & 2,57 \\
\hline & En cas de deuil & 21 & $0 \mathrm{a}$ & 0 & 79 & $0 \mathrm{a}$ & 0 & 107 & $7,48 \mathrm{a}$ & 4,98 \\
\hline & Oiseaux vicieux & 21 & $0 \mathrm{a}$ & 0 & 78 & $24,36 a$ & 9,53 & 107 & $13,08 a$ & 6,39 \\
\hline & En arrêt de ponte & 21 & $\mathrm{Ob}$ & 0 & 78 & $32,05 a$ & 10,36 & 107 & $22,43 a$ & 7,9 \\
\hline
\end{tabular}

$\%$ : Fréquence ; IC : Intervalle de confiance ; les pourcentages de la même ligne suivis des lettres différentes diffèrent significativement au seuil de $5 \%$; $\mathrm{N}$ : effectif total

Tableau 7 : Mortalité et morbidité des canards de barbarie

\begin{tabular}{|c|c|c|c|c|c|c|c|c|c|c|}
\hline \multirow{2}{*}{ Variables } & & \multicolumn{3}{|c|}{ Zone de dépression } & \multicolumn{3}{|c|}{ Zone des pêcheries } & \multicolumn{3}{|c|}{ Zone des terres de barre } \\
\hline & & $\mathrm{N}$ & $\%$ & $\mathrm{IC}$ & $\mathrm{N}$ & $\%$ & $\mathrm{IC}$ & $\mathrm{N}$ & $\%$ & $\mathrm{IC}$ \\
\hline \multirow{3}{*}{$\begin{array}{l}\text { Age auquel le taux de } \\
\text { mortalité est élevé }\end{array}$} & Avant sevrage & 21 & $100 a$ & 0 & 81 & $88,89 a$ & 6,84 & 107 & $91,59 a$ & 5,26 \\
\hline & Entre sevrage et début de la ponte & 21 & $0 b$ & 0 & 81 & $14,81 \mathrm{~b}$ & 7,74 & 107 & $28,04 a$ & 8,51 \\
\hline & Après âge de la 1ère ponte de la cane & 21 & $\mathrm{Ob}$ & 0 & 81 & $16,05 \mathrm{a}$ & 7,99 & 107 & $26,17 a$ & 8,33 \\
\hline \multirow{3}{*}{ Taux de morbidité } & Avant sevrage & 21 & $95,24 a$ & 9,11 & 81 & $50,62 \mathrm{c}$ & 10,89 & 107 & $70,09 b$ & 8,68 \\
\hline & Entre sevrage et début de la ponte & 21 & $0 \mathrm{a}$ & 0 & 81 & $3,7 a$ & 4,11 & 107 & $0,93 a$ & 1,82 \\
\hline & Après âge de la 1ère ponte de la cane & 21 & $0 \mathrm{a}$ & 0 & 81 & $4,94 a$ & 4,72 & 107 & $1,87 a$ & 2,57 \\
\hline \multirow{3}{*}{ Causes de la morbidité } & Coccidiose & 4 & Oa & 0 & 11 & $9,09 a$ & 16,99 & 28 & $3,57 a$ & 6,87 \\
\hline & Newcastle & 4 & $\mathrm{Ob}$ & 0 & 11 & $\mathrm{Ob}$ & 0 & 28 & $67,86 a$ & 17,3 \\
\hline & Maladie de Marek & 4 & $100 a$ & 0 & 11 & $90,91 \mathrm{a}$ & 16,99 & 28 & $28,57 \mathrm{~b}$ & 16,73 \\
\hline
\end{tabular}

$\%$ : Fréquence ; IC : Intervalle de confiance ; les pourcentages de la même ligne suivis des lettres différentes diffèrent significativement au seuil de $5 \%$; $:$ effectif total 
Traitement des canards locaux et origine des médicaments: Le tableau 8 présente le traitement médical des canards de Barbarie et l'origine des médicaments utilisés sur ces oiseaux. Contrairement aux éleveurs des terres de barre, ceux des pêcheries et de dépression, n'administrent aucun traitement (traditionnel ou moderne) aux canards en cas de pathologies $(p<0,05)$. Les éleveurs qui ne donnent aucun traitement aux oiseaux dans les trois zones agro-écologiques avaient pour raisons principales: le manque de moyens, la rusticité des canards, la sous information sur le suivi sanitaire et la bonne croissance des oiseaux malgré l'absence de suivi $(p>0,05)$. Lorsqu'il y a traitement, les produits utilisés sont dans la majorité des cas, par auto médication, surtout dans les zones des pêcheries $(40,24 \%)$ et des terres de barre $(50,47 \%)$. Ces produits peuvent être des déparasitants, des antibiotiques, des complexes vitaminés ou des vaccins.

Tableau 8 : Traitement des canards de Barbarie et origine des médicaments

\begin{tabular}{|c|c|c|c|c|c|c|c|c|c|c|}
\hline \multirow{2}{*}{ Variables } & & \multicolumn{3}{|c|}{ Zone de dépression } & \multicolumn{3}{|c|}{ Zone des pêcheries } & \multicolumn{3}{|c|}{ Zone des terres de barre } \\
\hline & & $\mathbf{N}$ & $\%$ & IC & $\mathbf{N}$ & $\%$ & IC & $\mathbf{N}$ & $\%$ & IC \\
\hline \multirow{3}{*}{$\begin{array}{l}\text { Types de } \\
\text { Traitement }\end{array}$} & Pas de traitement & 22 & $63,64 a$ & 20,1 & 84 & $46,43 a$ & 10,67 & 106 & $18,87 b$ & 7,45 \\
\hline & Traitement traditionnel & 22 & ob & 0 & 84 & $11,9 b$ & 6,92 & 106 & $36,79 a$ & 9,18 \\
\hline & Traitement moderne & 22 & $36,36 \mathrm{~b}$ & 20,1 & 84 & $46,43 b$ & 10,67 & 106 & $63,21 a$ & 9,18 \\
\hline \multirow{4}{*}{$\begin{array}{l}\text { Causes du } \\
\text { manque de } \\
\text { traitement }\end{array}$} & Manque de moyens & 5 & $42,11 a$ & 43,28 & 35 & $37,33 a$ & 16,02 & 56 & $28,41 a$ & 11,81 \\
\hline & Pas de maladie & 5 & $10,53 a$ & 26,9 & 35 & $4 a$ & 6,49 & 56 & $4,55 a$ & 5,46 \\
\hline & Sous information & 5 & $21,05 a$ & 35,73 & 35 & $12 a$ & 10,77 & 56 & $3,41 \mathrm{a}$ & 4,75 \\
\hline & Croissance rapide des cannetons & 5 & $26,32 a$ & 38,6 & 35 & $46,67 a$ & 16,53 & 56 & $63,64 a$ & 12,6 \\
\hline \multirow{5}{*}{$\begin{array}{l}\text { Produits utilisés } \\
\text { fréquemment } \\
\text { dans les élevages }\end{array}$} & Déparasitants & 22 & $22,73 a$ & 17,51 & 84 & $38,1 \mathrm{a}$ & 10,39 & 104 & $42,31 a$ & 9,5 \\
\hline & Vaccins & 22 & $4,55 b$ & 8,71 & 84 & $11,9 b$ & 6,92 & 104 & $23,08 a$ & 8,09 \\
\hline & Antibiotiques & 22 & $27,27 b$ & 18,61 & 84 & $29,76 b$ & 9,78 & 104 & $61,54 a$ & 9,35 \\
\hline & Complexes vitaminés & 22 & $13,64 b$ & 14,34 & 84 & $38,1 a$ & 10,39 & 105 & $51,43 a$ & 9,56 \\
\hline & Autres & 22 & Oa & 0 & 84 & $2,38 a$ & 3,26 & 107 & $2,8 \mathrm{a}$ & 3,18 \\
\hline \multirow{2}{*}{$\begin{array}{l}\text { Origine des } \\
\text { produits utilisés }\end{array}$} & Auto médication & 22 & $4,55 b$ & 8,71 & 82 & $40,24 a$ & 10,61 & 107 & $50,47 a$ & 9,47 \\
\hline & Prescription vétérinaire & 22 & $36,36 a$ & 20,1 & 82 & $18,29 a$ & 8,37 & 106 & $34,91 \mathrm{a}$ & 9,07 \\
\hline
\end{tabular}

\section{DISCUSSION}

Statut des éleveurs et fonctions socio-économique, culturelle et cultuelle de l'élevage de canard de Barbarie: Les niveaux d'instruction, la situation matrimoniale, le nombre moyen d'enfants et l'âge des éleveurs ne sont pas différents d'une zone agroécologique à une autre. Les proportions obtenues pour chacune de ces caractéristiques des élevages sont proches de celles obtenues chez les poulets locaux au Sud du Bénin (Youssao et al., 2013), au SudOuest du Nigeria (Oguntundji et al., 2014) et dans l'Etat de Ogun au Nigéria (Adeyemi et al., 2008). Des situations semblables à celle de notre étude sur la situation matrimoniale et le niveau d'instruction sont également rapportées par Alfred et Agbédé. (2012) au Philippine, Adeyemi et al. (2008) et Baruwa et al. (2018) au Nigéria sur les activités d'élevage du canard de barbarie. A l'issue de notre étude, il ressort que dans les zones des pêcheries et des terres de barre, l'élevage des canards de Barbarie est pratiqué majoritairement par les hommes alors que dans la zone de dépression les femmes sont dominantes. Cette différence, en faveur des femmes dans la zone de dépression, s'explique par leur marginalisation dans le secteur agricole en général dans l'élevage des espèces, autres que le canard, en particulier (Akpla, 2013 ; FAO, 2015.). D'autres auteurs ont rapporté les mêmes observations dans différentes études sur les volailles à savoir : Youssao et al. (2013) sur les poulets locaux Gallus-gallus élevés au Bénin, Sanfo et al. (2007) sur les élevages de pintades au Burkina-Faso et Katchouang et al. (2015) au Cameroun sur les élevages de cailles. La proportion de commerçants et éleveurs de canard est plus élevée dans les zones de dépression et de pêcherie que dans les terres de barre. Cette différence s'explique par la dominance des éleveurs de la zone des terres de barre qui ont comme activités principale, l'agriculture. Les éleveurs des différentes zones agro écologiques mènent plusieurs types d'activités. Ils sont des agriculteurs, des artisans, des ménagères, des salariés et des éleveurs professionnels dans les trois zones étudiées. L'élevage dans la plupart des familles est alors une activité secondaire qui procure aux propriétaires des moyens usuels pour régler les petits problèmes. De même, la 
diversité des activités des éleveurs observée dans les trois zones de cette étude atteste que l'élevage n'est pas effectivement le seul moyen de subsistance des familles. Des résultats similaires sont rapportés sur l'élevage des poulets locaux au Bénin (Youssao et al., 2013) et sur l'élevage des canards au Nigeria (Baruwa et al., 2018). Les canards de barbarie sont élevés pour le prestige surtout dans les zones agro écologiques des pêcheries et des terres de barre. Dans ces deux zones, les canards sont considérés comme des oiseaux de grande valeur sur les plans culturel et cultuel et sont utilisés lors des cérémonies traditionnelles (dot, sacrifice etc.). La même pratique est également rapportée dans les communes d'Akpro-misséréte et de Kétou dans la zone des terres de barre au sud du Bénin (Akpla, 2013), au Sud-Ouest du Nigeria (Oguntundji, 2014) et au Philippine (Alfred et Agbédé, 2012).

Comparaison des performances d'élevage entre le canard et le poulet: Dans les trois zones agro écologiques de l'étude, les éleveurs ont déclaré que, les canards de barbarie sont peu exigeants en matière d'habitat par rapport aux poulets. Cette faible exigence par rapport aux poulets s'explique par leur plus grande rusticité (Amanidja et al., 2018) du fait qu'ils résistent mieux aux pathologies aviaires et parviennent à vivre et à se reproduire de façon satisfaisante malgré les carences alimentaires auxquelles ils peuvent être soumis (Nkidiaka et Huart, 2013). Baruwa et al. (2018) rapportent également que ces oiseaux ne nécessitent pas un grand investissement surtout par rapport à leur habitat. L'élevage des canards au Sud-Bénin est de type familial avec un système d'élevage extensif. Les enquêtés dans les trois zones agro-écologiques signalent que les canards de barbarie ont les mêmes exigences sur le plan alimentaire que les poulets locaux. Selon les enquêtés, ces deux espèces sont abattues au-delà de 5 mois d'âge et les canards de barbarie présentent plus de graisse que les poulets. Très souvent, ces oiseaux sont considérés comme une volaille grasse à cause de la quantité de gras abdominal et de l'ensemble "peau+ gras souscutané » qui sont plus importantes chez les palmipèdes dont la teneur en lipides est proche voire inférieure à celui des poulets (Chartin et al., 2006 ; Baeza et al., 2013).

Caractéristiques des exploitations des canards de Barbarie au Sud-Bénin : Les exploitations d'élevage de canard des trois zones agro-écologiques de la présente étude ont été créées entre 2000 et 2016. Ces élevages sont jeunes et la plupart ont été mis en place entre 2004 et 2013. L'avènement de la grippe aviaire dans cette période a probablement orienté les éleveurs de poulets vers d'autres espèces plus rustiques comme le canard. Par ailleurs, il a été constaté que l'élevage du canard de barbarie dans les trois zones agroécologiques est souvent accompagné de celui du poulet surtout dans la zone de dépression et de la pintade dans les zones des pêcheries et des terres de barre. Oguntundji et Ayorinde (2015) dans une étude sur la production du canard au Nigéria, ont fait cas de la préférence des éleveurs vis-à-vis d'autres espèces de volaille au détriment du canard de barbarie. Cette association de l'élevage du canard à d'autres volailles est observée par Baruwa et al. (2018) au Nigéria et Amanidja et al. (2018) en Côte d'lvoire. Les canards sont très souvent identifiés par les fermiers grâce à leur plumage ou à des ficèles attachés aux ailes dans les trois zones. Les oiseaux n'ont pas de bagues ni de numéro pour les retrouver et suivre leur évolution dans le cheptel, ce qui freine le contrôle de la production (Mugumaarhahama et al., 2016). L'un des avantages liés à l'élevage du canard de barbarie dans les zones d'étude est la prolificité de la cane mais l'inexistence de marchés d'écoulement est la difficulté la plus rencontrée par les éleveurs de cette espèce. Ces résultats confirment ceux d'autres études réalisées sur les canards de barbarie par Amanidja et al. (2018) en Côte d'Ivoire et par Baruwa et al. (2018) au Nigéria. La taille moyenne des effectifs de canards dans les exploitations est de 16 oiseaux dans la zone de dépression, 30 dans la zone des pêcheries et de 22 dans la zone des terres de barre avec la zone des pêcheries qui a le nombre de canetons à l'éclosion et la mortalité au sevrage les plus élevés. Ce taux élevé de mortalité enregistré dans la zone des pêcheries est peut-être dû à l'humidité importante observée dans cette zone. Toutefois, l'effectif du cheptel dans les élevages de canard de barbarie de nos différentes zones est plus important que celui enregistré par Baruwa et al. (2018) au Nigéria et Amanidja et al. (2018) en Côte d'Ivoire qui tourne autour de dix (10) canards par exploitation tout âge confondu

Mode d'élevage et exploitation des oiseaux dans le Sud-Bénin : Dans la présente étude, la quasi-totalité des éleveurs de la zone de dépression pratique l'élevage en divagation contre la moitié des éleveurs dans les zones agro-écologiques des pêcheries et des terres de barre. Les oiseaux sont livrés à eux-mêmes et ne sont pas suivis surtout dans la zone de dépression. Ces canards sortent le matin à la recherche d'aliments et ne reviennent que le soir pour recevoir des restes de 
cuisine et des produits de récolte. Toutefois, dans les zones des pêcheries et des terres de barre, plus de la moitié des éleveurs donnent un complément d'aliment (provende) aux oiseaux. Ces éleveurs prennent mieux soin des oiseaux sur le plan alimentaire parce qu'en dehors de la vente, ils consomment les canards et les utilisent dans diverses cérémonies traditionnelles comme la dot, les sacrifices et autres. Dans la commune d'Akpro-missérété dans la zone agroécologique des terres de barre, les éleveurs élèvent ces oiseux parce qu'ils ne sont pas exigeants en termes d'habitat et d'alimentation (Akpla, 2013). Les mêmes observations ont été faites par Oguntundji et Ayorinde (2015), Adeyemi et al. (2008) et Baruwa et al. (2018) au Nigéria. Aussi, en Côte-d'Ivoire, les canards sont élevés dans un système familial extensif en divagation où les oiseaux parcourent le village à la recherche d'aliment toute la journée (Amanidja et al., 2018). Les éleveurs produisent du canard pour la viande pour la vente ou l'autoconsommation (Adeyemi et al., 2008 ; Oguntundji et Ayorinde, 2015 ; Baruwa et al., 2018 et Amanidja et al., 2018). La reproduction chez les canards de barbarie est naturelle et généralement, le père n'est pas connu, contrairement à la mère. Dans certains élevages du Sud-Ouest du Nigeria, seule la mère est également connue et l'incubation des œufs est faite sous cane (Oguntundji et Ayorinde, 2015).

\section{CONCLUSION}

L'étude sur les caractéristiques de l'élevage de canard de barbarie dans les trois zones agro-écologiques du Sud du Bénin a révélé que, dans la zone de dépression, cette activité est pratiquée majoritairement par les femmes alors que dans les deux autres zones ce sont les hommes qui dominent. Dans la zone de dépression, les canards sont élevés en divagation et l'aliment distribué est généralement composé de céréales, des restes de récolte et de cuisine. Dans cette zone, les oiseaux sont élevés pour la vente et aucun soin particulier n'est prodigué aux canards. Dans les zones des pêcheries et des terres de barre, les canards de barbarie sont élevés en divagation et

\section{REMERCIEMENTS}

Les auteurs remercient le Ministère de l'Enseignement Supérieur de la Recherche Scientifique (MESRS) pour le financement de ces travaux de recherche intitulé :
Mortalité et suivi sanitaire : Dans les trois zones agro écologiques de notre étude, les cas de mortalité et de morbidité sont le plus souvent observés avant le sevrage chez les canetons de moins de 8 semaines d'âge. Les causes de ces cas de mortalité et de morbidité sont les maladies, les accidents domestiques et la saison. L'enregistrement de la forte mortalité pendant le sevrage s'explique par le manque de suivi lié aux conditions d'élevage précaires qu'offrent les fermiers aux canetons. Après éclosion, ces canetons devront faire face aux nombreux défis environnementaux (Amanidja et al., 2018). Adeyemi et al. (2008), Oguntundji et Ayorinde (2015) et Baruwa et al. (2018) rapportent la même situation sur la mortalité et l'état de morbidité observés chez les canetons au Nigéria. De même, Sanfo et al. (2007), Youssao et al. (2013) et Katchouang et al. (2015) rapportent également les taux élevés de mortalité pendant les premières semaines de la vie, respectivement dans les élevages traditionnels de pintades au Burkina-Faso, de poulets locaux au Bénin et de cailles au Cameroun. Le suivi sanitaire des canards dans les trois zones de notre étude est moins rigoureux surtout dans la zone de dépression et se limite chez la plupart des éleveurs à une automédication. Dans la commune d'AkproMissérété située dans la zone des terres de barre, ces oiseaux ne bénéficient d'aucun soin particulier mais arrivent à vivre et à se reproduire (Akpla, 2013).

certains des éleveurs mettent à la disposition des oiseaux un abri plus parcours. En dehors des restes de récolte et de cuisine, les canards de barbarie reçoivent un complément alimentaire (provende). Ils sont utilisés pour la vente et l'autoconsommation surtout dans la zone des terres de barre. Sur le plan sanitaire, les oiseaux sont traités généralement par auto médication. II est remarqué que des efforts restent à fournir pour rendre dynamique l'élevage du canard de barbarie au Sud-Bénin. Pour ce faire, la maitrise des types d'élevage de canard dans ces zones serait salutaire pour le développement du secteur.

« Caractérisation morphologique et zootechnique de la population locale du canard de barbarie au SudBénin ». 


\section{REFERENCES BIBLIOGRAPHIQUES}

Adeyemi OA, Sobayo RA, Aluko FA, 2008. A survey of duck farming activities in Abeokuta metropolis of Ogun State, Nigerie. Nigeria Poultry Science Journal, 5: 23- 29.

Akpla MCK, 2013. Les fonctions socioculturelles du canard de Barbarie (Caïrina moschata) dans les communes d'Akpro-misséréte et de Kétou au sud du Bénin. www.slire.net/document

Alfred SDY, Agbede JO, 2012. Factors of influence of the production duck in South West Nigeria. African Agricultural Research Journal, 7: 34983505.

Amanidja BD, Komara M, Soro D, Loba A, Logboh EG, Atcho 0,2018 . Analyse diagnostique de l'élevage des Canards de race locale (Cairina Moschata Linnaeus, 1758) dans le Sud Forestier de la Côte d'Ivoire. European Scientific Journal, 14: 1857-7881.

Baeza E, Chartrin P, Bordeau T, Lessire M, Thoby JM, Gigaud V, Blanchet M, Alinier A, Leterrier C, 2013. Omega-3 polyunsaturated fatty acids provided during embryonic development improve the growth performance and welfare of Muscovy ducks (Cairinamoschata). Poultry Science, 96: 3176-3187.

Baruwa OI, Tijani AA, Alimi T, 2018. Determinants of technical efficiency in duck production in southwest Nigeria. Agricultura Tropica et Subtropica, 51: 113-120.

Boko CK., 2012. Salmonella enterica dans les mortalités de pintadeaux au Bénin : Etude de terrain, comparaison des souches, et activité antibactérienne des extraits de plantes locales. Thèse de doctorat en Santé et Productions Animales. Université de Liège (Ulg), 113p.

Chartrin P, Meteau K, Juin H, Bernadet MD, Guy G, Larzul C, Remignon H, Mourot J, Duclos MJ., Baeza E, 2006. Effects of Intramuscular Fat Levels on Sensory Characteristics of Duck Breast Meat. Poultry Science 85: 914-922.

Chrysostome C, 1995. Méthodologie de développement de la pintade au Benin. Thèse de doctorat. Sciences Agronomiques, Institut national Agronomique. Paris-Grignon, 190p et Annexes.

Dahouda M, 2009. Contribution à l'étude de l'alimentation de la pintade locale au Bénin, et perspectives d'améliorations à l'aide de ressources non conventionnelles. Thèse de doctorat en Santé et productions animales à L'Université de Liège. 174p.

DE (Direction de l'Élevage), 2016. Rapport annuel 2015. République du Bénin. 106 pp.

FAO, 2015. Secteur Avicole Bénin. Revues nationales de l'élevage de la division de la production et de la santé animales de la FAO. No. 10. Rome.

Hondonougbo PV, 2017: Caractérisations phénotypiques des populations de pintades (Numida meleagris) locales élevées au Bénin. Thèse de doctorat $(\mathrm{PhD})$. Université de Liège, Gembloux Agro-Bio Tech (Belgique), 130p.

Houessionon FJB, Youssao IAK, 2018. Evaluation des performances zootechniques des canards de barbarie (cairina moschata) au Sud-Bénin. Journée Scientifique du Centre Béninois de la Recherche Scientifique et de l'innovation du 26 au 28 Juin 2018 au Champ de Foire, Cotonou, Bénin.

Katchouang ASN, Djitie FK, Meutchieye F, Kana JR, Teguia A, 2015. Caractéristiques des élevages de caille (Coturnix sp) dans le département du Mfoundi, région du Centre, Cameroun. Livestock Research for Rural Development, 27: http://www.Irrd.org//rrd27/4/katc27077.htm

Mugumaarhahama $Y$, Ayagirwe RBB, Mutwedu VB, Sadiki JM, Baenyi $P$, Mushagalusa $A C$ Bisimwa EB, 2016. Caractérisation des systèmes de production de poule locale dans deux zones agro-écologiques du Sud-Kivu (République Démocratique du Congo). Livestock Research for Rural Development, 28:

http://www.lrrd.org//rrd28/1/mugu28007.html

Nkidiaka LO, Huart A, 2013. Reportage: La situation de l'élevage de volaille en RDC et à Kinshasa. Troupeaux et Cultures des Tropiques, 72-74.

Oguntundji AO, 2014. Taboos, superstitions, myths and stimas against duck production in south-west Nigeria. Wayamba Journal of Animal Science, 6: 998-1007.

Oguntunji AO. ET Ayorinde KL, 2015. Duck production in Nigeria: flock characteristics, management and mortality. Archiva Zootechnica, 18 (1): 27-40.

Programme d'action national d'adaptation aux changements climatiques du Bénin (PANABENIN), 2007. Convention-cadre des nations unies. 81pp 
Sanfo R, Boly H, Sawadogo L, Ogle B, 2007. Caractéristiques de l'élevage villageois de la pintade locale (Numida meleagris) au centre du Burkina Faso. Tropicultura, 25: 31-36.

SAS, 2013. SAS/STAT User's guide, vers, 9.4 Utilities, Cary, NC, USA, SAS Institute Inc.

Youssao IAK, Tougan UP, Ahounou SG, Houessionon BFJ, Koutinhouin B, 2013. Typology of local poultry breeding of Gallus gallus species in family poultry in Benin. International Journal of Agronomy and Agricultural Research, 3:1-13. 